\title{
Actually, Serial Template Satisfaction Does Predict Medial Coda Skipping in Reduplication
}

\author{
Sam Zukoff \\ Massachusetts Institute of Technology
}

\section{Introduction}

The pattern of "reduplicant-medial coda skipping" [henceforth coda skipping] is unattested as a mode of copying in reduplication. The term coda skipping refers to a language that permits codas in roots/bases (i.e. CV C.CV...), but disallows codas from appearing in medial position in a polysyllabic reduplicant (i.e. CV.CV-CV C.C V..., not *CVC.CV-CV[C.CV...). McCarthy, Kimper, \& Mullin (2012) [henceforth MKM] claim that Serial Template Satisfaction (STS), a framework proposed by MKM for analyzing reduplication within Harmonic Serialism (HS; see McCarthy 2000, 2010, a.o.), is unique in excluding this copying pattern, which is predicted by most other theories of reduplication, notably including Base-Reduplicant Correspondence Theory (BRCT; McCarthy \& Prince 1995). STS's apparent advantage in this domain is taken as a point in favor of STS over BRCT.

In this paper, I show that this claim cannot be sustained. STS, just like BRCT, must choose between allowing the unattested coda skipping pattern and disallowing the attested pattern of onset skipping, wherein complex onsets are permitted in roots/bases (i.e. $\overline{C C V}$...) but disallowed in reduplicants (i.e. C]V-CCV..., not $* \mathrm{CCV}-\mathrm{CCV}$...). In both frameworks, the mechanics that would permit onset skipping straightforwardly predict coda skipping, as well. Therefore, this empirical domain does not, in fact, provide a means of distinguishing between STS and BRCT.

\section{The mechanics of STS, and the (supposed) non-generation of medial coda skipping}

In STS, surface patterns of reduplicant shape are generated by a schema consisting of three elements. First, reduplicative morphemes are lexical entries that consist of empty prosodic structure (e.g. foot, syllable, or mora, per the Prosodic Morphology Hypothesis; McCarthy \& Prince 1986 [1996]). Second, there is a family of constraints, HEADEDNESS(X), that require prosodic constituents (of type $\mathrm{X}$ ) to have heads (of type $\mathrm{X}-1)$. Lastly, there is an operation $\operatorname{Copy}(\mathbf{X})$ (penalized by the constraint ${ }^{*} \operatorname{COPY}(\mathrm{X})$ ) that copies a contiguous string of constituents of type $\mathrm{X}$ from the base, for the purposes of satisfying HEADEDNESS (for the relevant prosodic category). There is an alternative operation which can be employed to satisfy HEADEDNESS, Insert(X), which inserts an empty prosodic category of the type that can serve as a head for the empty template; for example, an empty syllable can be inserted to provide a head to an empty foot. ${ }^{1}$

The surface shape of the reduplicant thus depends on (i) the type of underlying prosodic template the morpheme has, and (ii) the ranking of the various constraints of the HEAdEDNESs(X) and ${ }^{*} \operatorname{CoPY}(\mathrm{X})$ constraint families. As in HS generally, constraint ranking determines the order in which operations apply to the input. That ordered derivation leads to distinctly different shape characteristics of the surface reduplicant.

The tableaux in (1) and (2) demonstrate the two primary ways of filling a foot-sized reduplicative template. In Manam (1), which has a right-edge bimoraic foot template, the ranking FoOT-BINARITY $\gg$ * $\operatorname{COPY}(\sigma)$ forces syllable-copying at Step 1 , since it is impossible to satisfy Foot-BINARITY through a single application of the Insert operation. ${ }^{2}$

\footnotetext{
* I would like to thank Adam Albright, Edward Flemming, Donca Steriade, and the audience at AMP 2016. All mistakes and infelicities are of my own doing.

1 Based on the operation-to-constraint logic of HS, this operation should be accompanied by a (freely-rankable) constraint *INSERT(X). MKM do not employ such a constraint (nor explore its potential consequences), though they allow that it might exist.

2 It is a tacit assumption of MKM that, if the string laga were copied as a string of segments rather than a string of 
(1)

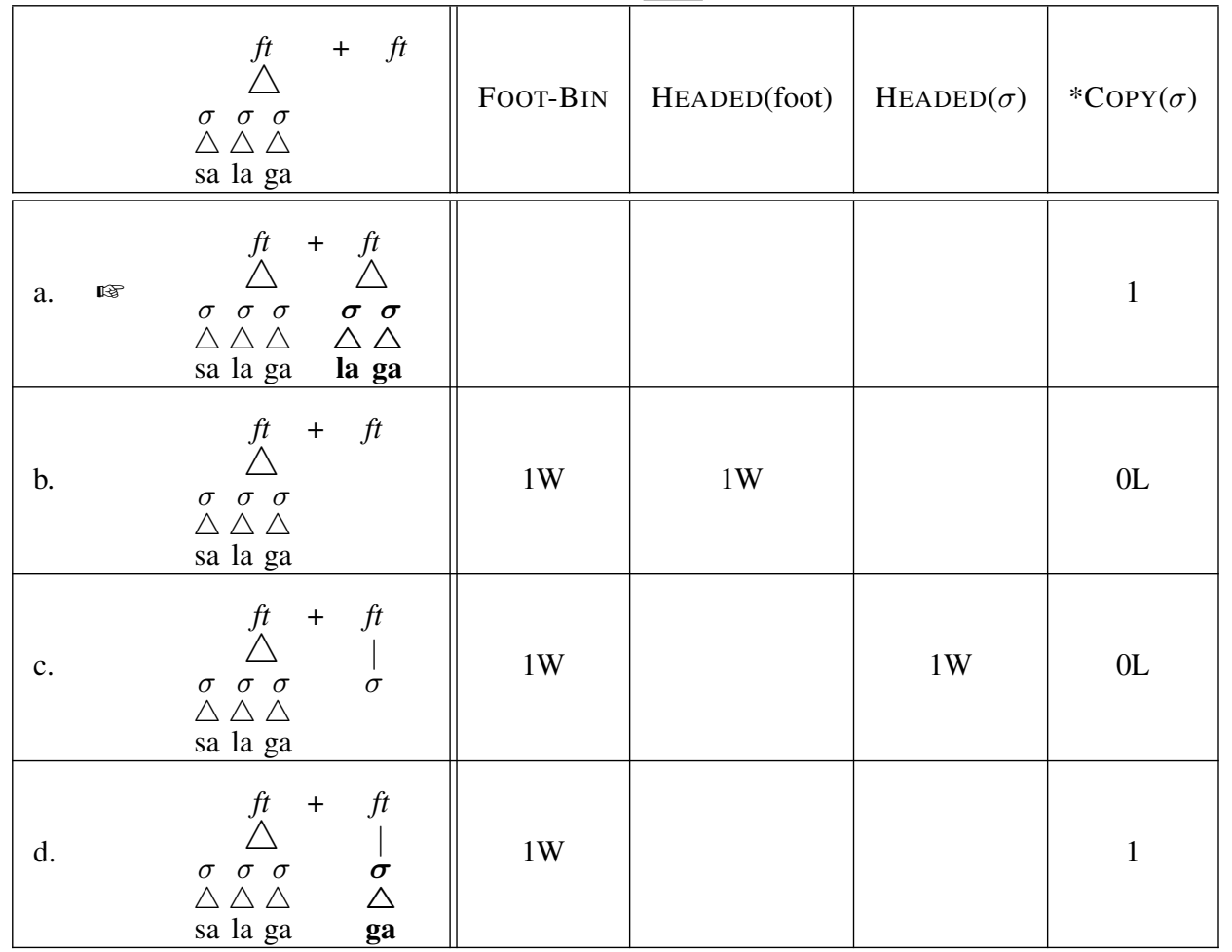

In Balangao (2), which has a left-edge disyllabic foot template, the ranking $* \operatorname{COPY}(\sigma) \gg \operatorname{HEADED-}$ NESS(foot) $\gg$ FOOT-BINARITY favors two-step syllable-insertion to satisfy FOOT-BINARITY; the ranking FOOT-BINARITY $\gg \operatorname{HEADEDNESS}(\sigma) \gg * \operatorname{COPY}(\mathrm{seg})$ then generates segment-copying to fill the newly created empty syllables.

(2) Segment copying in Balangao: taynan $\rightarrow$ tayna-taynan

(adapted from MKM:184-186)

i. $\quad$ Step 1: Syllable-insertion

\begin{tabular}{|c|c|c|c|c|c|c|}
\hline & 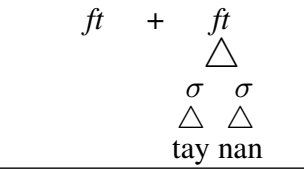 & $* \operatorname{COPY}(\sigma)$ & $\mathrm{HD}$ (foot) & FOOT-BIN & $\operatorname{HD}(\sigma)$ & $* \operatorname{COPY}(\mathrm{seg})$ \\
\hline a. & 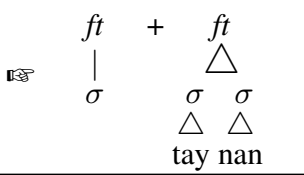 & & & 1 & 1 & \\
\hline b. & 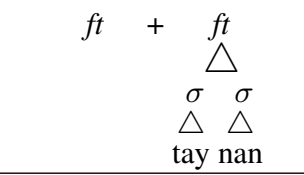 & & $1 \mathrm{~W}$ & 1 & $0 \mathrm{~L}$ & \\
\hline c. & 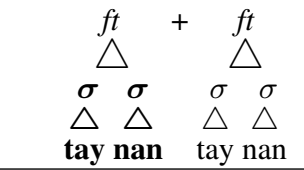 & $1 \mathrm{~W}$ & & $0 \mathrm{~L}$ & $0 \mathrm{~L}$ & \\
\hline
\end{tabular}

syllables, that they could not automatically be parsed into syllables that are themselves parsed into the foot in the same derivational step. That is to say, HEADEDNESs(foot) can never be satisfied by applying the Copy(seg) operation; more generally, Headedness(X) can never be satisfied by applying the $\mathbf{C o p y}(\mathbf{X}-2)$ operation. 
ii. Step 2: Syllable-insertion (again)

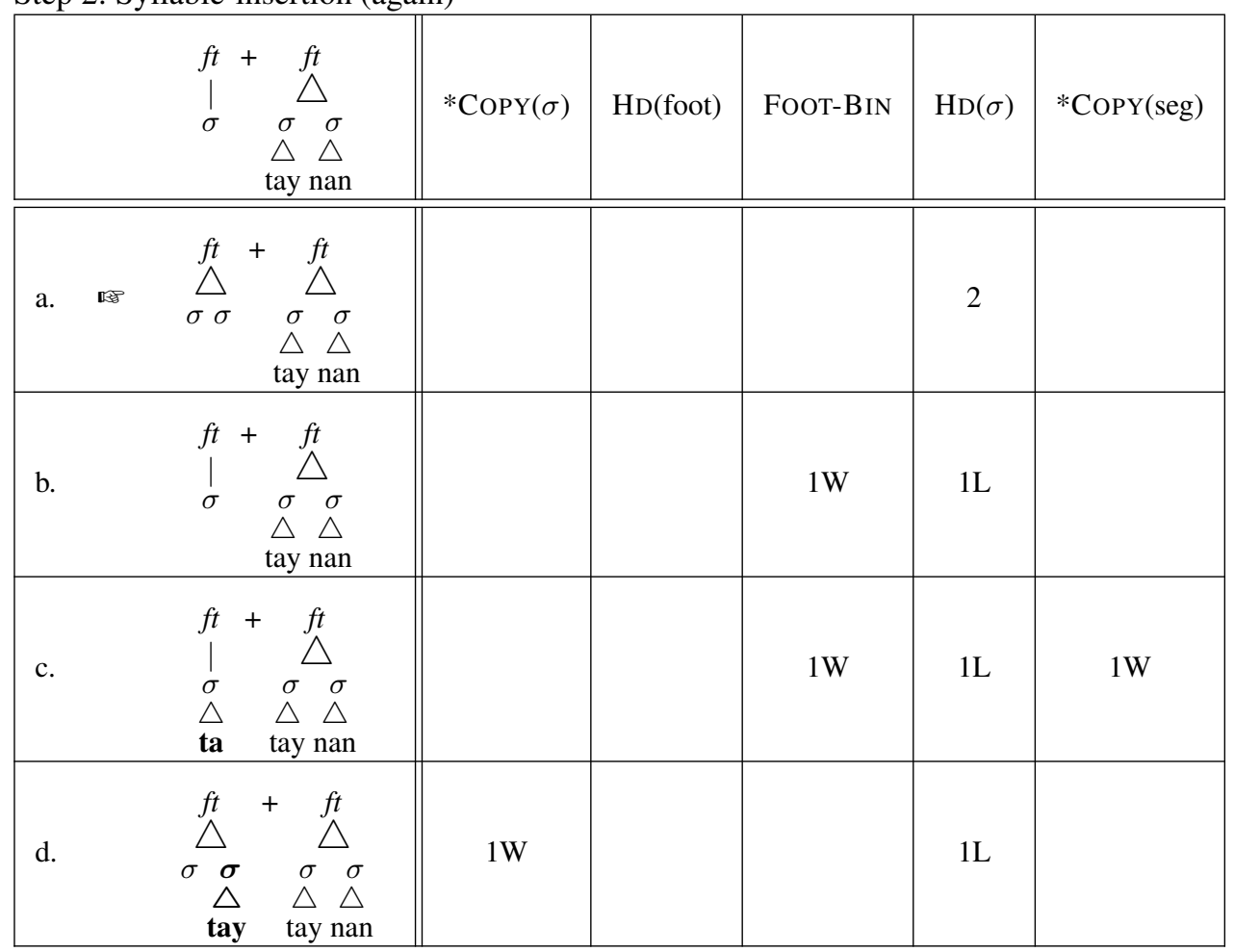

iii. Step 3: Segment-copying

\begin{tabular}{|c|c|c|c|c|c|}
\hline 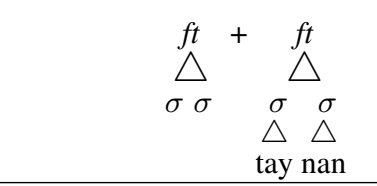 & $* \operatorname{CoPY}(\sigma)$ & HD(foot) & FOOT-BIN & $\operatorname{HD}(\sigma)$ & *COPY(seg) \\
\hline 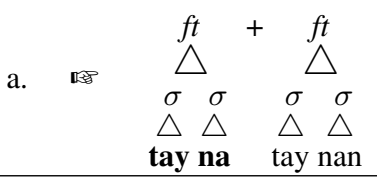 & & & & & 1 \\
\hline 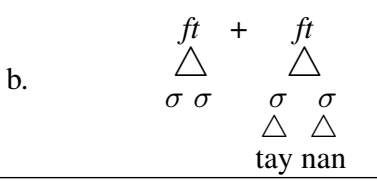 & & & & $2 \mathrm{~W}$ & OL \\
\hline 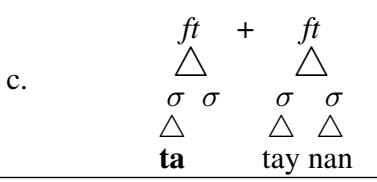 & & & & $1 \mathrm{~W}$ & 1 \\
\hline
\end{tabular}

In a language like Balangao which obtains its reduplicated segments via segment copying (after inserting empty syllable nodes into a foot template), the copy operation has some amount of freedom in the string of segments that it copies. While it must be a contiguous string, that string can terminate at any point. That is to say, when filling the empty syllables in (2) through segment copying, there are a number of possible candidates, including those shown in (3): 

a. *ta.i-tay.nan
b. *ta.yn-tay.nan
c. $\overline{\text { tay.na-tay.nan }}$
d. * *ay.nan-tay.nan

Since the Copy(seg) operation is blind to the number of segments copied (MKM:180ff), these candidates are all equivalent with respect to the constraints included in the preceding tableaux. Since there are no built in pressures for smaller reduplicants (given the same prosodic structure) in STS - contrary to a number of "a-templatic" approaches to partial reduplication in BRCT (Gafos 1998, Hendricks 1999, Riggle 2006, among many others) - considerations of overall size will not be relevant in selecting between these candidates. Instead, the choice between the competing segment-copying candidates will be made purely by phonotactics.

To choose attested tay.na-tay.nan over the other possibilities, we need only invoke basic syllabic wellformedness constraints: *ta.i-tay.nan violates ONSET/NOHIATUS; *ta.yn-tay.nan violates constraints against consonantal nuclei (e.g. HNUC; Prince \& Smolensky 1993/2004); and, most relevantly, *tay.nan-tay.nan violates NOCODA twice, as opposed to just once in the desired output tay.na-tay.nan. Since the winner does violate NoCODA, Balangao must have the ranking ONSET/NoHiatus, HNUC $\gg$ NoCODA, a ranking which seems consistent with the general phonology of the language (as codas are generally allowed). Nonetheless, the activity of NoCODA might lead one to expect that a candidate that altogether eschews reduplicant codas, e.g. *ta.na-tay.nan, should be preferred to the actual winner, which violates NoCODA once. However, this candidate is unavailable (at this step in the derivation; though, crucially, it is available at later stages of the derivation - see below) due to the nature of the Copy operation, as it would have required copying a discontiguous string of segments.

Based on a survey of disyllabic partial reduplication in Australian and Austronesian languages, MKM find that there are no languages that have a pattern equivalent to this unavailable candidate, *ta.na-tay.nan. They refer to this pattern as the "CVCV" pattern, as it derives a CVCV string even when additional contiguous segmental material that could expand the string is present in the base. This pattern, illustrated in (4a), can be contrasted with the " $\sigma \mathrm{CV}$ " pattern (4b), attested by Balangao and many others, and the " $\sigma \mathrm{CVC}$ " pattern (4c), which MKM claim to also be unattested (and also underivable in STS).

\begin{tabular}{|c|c|c|c|}
\hline \multicolumn{4}{|c|}{ Predicted reduplicant shape patterns (hypothetical) } \\
\hline Root & a. CVCV (unattested) & b. $\sigma \mathrm{CV}$ (attested) & c. $\sigma \mathrm{CVC}$ (unattested) \\
\hline mele & mele-mele & mele-mele & mele-mele \\
\hline kalan & kala-kalan & kala-kalan & kalan-kalan \\
\hline paltiru & pati-paltiru & palti-paltiru & paltir-paltiru \\
\hline mikartu & mika-mikartu & mika-mikartu & mikar-mikartu \\
\hline nampalu & napa-nampalu & nampa-nampalu & nampal-nampalu \\
\hline
\end{tabular}

The CVCV pattern is easily derivable in BRCT, due to its fully parallel global evaluation mechanics: the CVCV candidate is available (since all candidates are available), and, given the ranking NOCODA $\gg$ CONTIGUITY-BR ('the reduplicant must correspond to a contiguous string in the base'), the $\mathrm{CVCV}$ candidate is harmonically superior to corresponding candidates with one or two codas. Given the unavailabil-ity of this candidate in STS (at the point of segment copying), MKM claim that STS is incapable of deriving the $\mathrm{CVCV}$ pattern. Given that the $\mathrm{CVCV}$ pattern is apparently unattested, ${ }^{3}$ this constitutes a point in favor of STS relative to BRCT, and BRCT overgenerates.

This claim rests on the assumption that there is no other means in the STS framework of deriving such a pattern. This assumption is demonstrably false. Once the pattern of "onset skipping" reduplication (MKM:§6.6) is fully reckoned with, it is clear that the mechanics that are required in order to derive such a pattern predict that the CVCV pattern can be derived in subsequent derivational steps. This is to be expected, because onset skipping and medial coda skipping are formally identical, modulo the specific markedness trigger, *COMPLEX in the former case, NOCODA in the latter.

3 It should be noted that their survey was based on only two language families, Australian and Austronesian. While extensive and suggestive, this survey is by no means exhaustive. 


\section{Onset skipping in STS predicts coda skipping in STS}

While reduplicant-medial coda skipping may be unattested, there is at least one other type of reduplicantinternal skipping pattern whose existence is not disputed. In a number of Indo-European languages (Steriade 1988, a.o.), as well as in Klamath (Barker 1964), and perhaps others, the second member of a base-initial consonant cluster fails to be copied into the reduplicant (see also Fleischhacker 2005). MKM illustrate this sort of pattern with data from Sanskrit, as shown in (5):

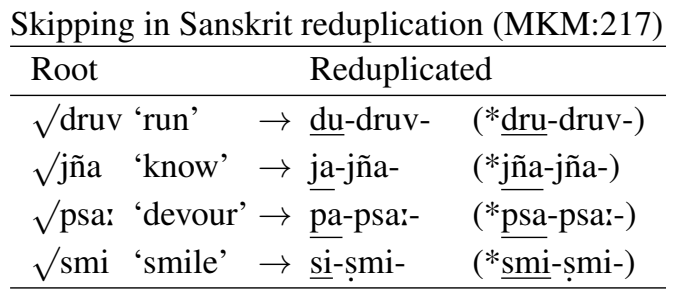

In this pattern, the reduplicant corresponds to a discontiguous string of segments in the base. Since copying in STS is limited to contiguous strings, this pattern cannot be generated by a single derivational step. The reduplicant cannot be built up through multiple steps of partial copying. Copying just the initial consonant, as in a candidate $/ \sigma$-druv- $/ \rightarrow \underline{d}$-druv-, would fail to satisfy $\operatorname{HeAdedness}(\sigma)$, and simultaneously introduce a phonotactically illegal initial geminate. Copying just the vowel, $/ \sigma$-druv- $/ \rightarrow$ $\underline{u}$-druv-, would satisfy HEADEDNESS $(\sigma)$ (though violating the constraint on locality of copying, which MKM name COPY-LOCALLY; MKM:181), and thus not motivate additional copying at the subsequent step. Copying could in theory be triggered by other markedness constraints, e.g. ONSET, but the fact that onsetless initial syllables are allowed in Sanskrit rules out this possibility.

Instead of multiple partial copying, this pattern can only be derived through full copying plus subsequent deletion. This requires a straightforward implementation of the emergence of the unmarked (TETU; McCarthy \& Prince 1994, 1995). Complex onsets are permitted in roots: MAXRooT $\gg *$ COMPLEX; but must be simplified (through deletion) in affixes: *COMPLEX $\gg$ MAXAFFIX (or a general MAX constraint). This derivation is shown in (6):

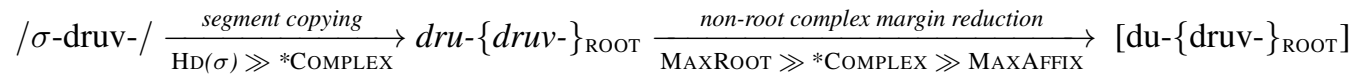

The introduction of TETU mechanics into the STS system allows for the possibility of reductions of numerous other sorts in post-copying derivational steps. It is a trivial extension to see how this can derive the previously impossible medial coda skipping (CVCV) pattern. All that is required is to replace *COMPLEX with NOCODA, as shown in (7):

$$
/\{\sigma \sigma\}_{f t} \text {-paltiru } / \underset{\mathrm{HD}(\sigma) \gg \text { NoCODA }}{\stackrel{\text { segment copying }}{\longrightarrow}} \text { palti- }\{\text { paltiru }\}_{\mathrm{ROOT}} \frac{\text { non-root coda reduction }}{\mathrm{MAXRT} \gg \text { NoCODA } \gg \mathrm{MAXAFX}} *\left[\text { pati- }\{\text { paltiru }\}_{\mathrm{ROOT}}\right]
$$

Prior to their discussion of Sanskrit-type onset skipping effects, MKM admit that reduplicant-specific constraints could induce skipping effects of various sorts - for example, a NoCODA constraint specifically indexed to the reduplicant could generate the medial coda skipping pattern - but the existence of such constraints was generally controversial (MKM:192). They also show that apparent cases of the skipping could arise authentically in their system, but only if they were the result of a phonological process which was generally applicable in the language - they claim that Seediq represents such an example (MKM:189, 220-222). However, the TETU scenario in (7) makes use of neither reduplicant-specific constraints nor general phonological processes to derive medial coda skipping. There is no theory-internal reason (nor any obvious theory-external reason) to expect that this ranking should be disallowed while the one required to derive the onset skipping pattern is permitted. Therefore, it must be said that STS actually does freely predict medial coda skipping in reduplication. In order to maintain the claim that STS does not predict such a pattern, the TETU mechanics would have to be abandoned. This would leave the onset skipping pattern, and indeed numerous other well-established cases of the emergence of the unmarked in reduplication, without an explanation in STS, leading to a very serious under-generation problem. 


\section{Takeaway}

The failure of STS to properly account for the supposed absence of the reduplicant-medial coda skipping pattern is not what is at issue here. What is at issue is the validity of the claim that STS is superior to BRCT specifically because of its performance in the particular empirical domain of skipping effects. BRCT very clearly predicts that skipping patterns, including the reduplicant-medial coda skipping (CVCV) pattern, should exist. This paper has demonstrated that STS in fact makes exactly the same prediction. This means that this empirical domain is actually not probative in the comparison between the two competing theories. Furthermore, this removes one of the major claimed results of STS from consideration in this comparison. With this result nullified, coupled with concerns over MKM's interpretation of the reduplication-phonology interaction data, and the tools required to capture other complex reduplication patterns (cf. Somerday 2015), it is unclear if STS is even viable in its current form, let alone a superior analytical framework to BRCT.

\section{References}

Barker, M. A. R (1964). Klamath Grammar. University of California Press, Berkeley.

Fleischhacker, Heidi Anne (2005). Similarity in Phonology: Evidence from Reduplication and Loan Adaptation. Phd dissertation, UCLA.

Gafos, Diamandis (1998). A-Templatic Reduplication. Linguistic Inquiry 29:3, 515-527.

Hendricks, Sean Q. (1999). Reduplication without Template Constraints: A Study in Bare-Consonant Reduplication. Phd dissertation, University of Arizona.

McCarthy, John J. (2000). Harmonic Serialism and Parallelism. Hirotani, Masako, Andries Coetzee, Nancy Hall \& Jiyung Kim (eds.), NELS 30: Proceedings of the North East Linguistic Society, GLSA, Amherst, MA, 501-524.

McCarthy, John J. (2010). An Introduction to Harmonic Serialism. Language and Linguistics Compass 4:10, 1001-1018.

McCarthy, John J. \& Alan Prince (1986 [1996]). Prosodic Morphology. Linguistics Department Faculty Publication Series 13.

McCarthy, John J. \& Alan Prince (1994). The Emergence of the Unmarked: Optimality in Prosodic Morphology. Gonzàlez, Mercè (ed.), NELS 24: Proceedings of the North East Linguistic Society, GLSA, Amherst, MA, 333379.

McCarthy, John J. \& Alan Prince (1995). Faithfulness and Reduplicative Identity. Beckman, Jill, Suzanne Urbanczyk \& Laura Walsh Dickey (eds.), University of Massachusetts Occasional Papers in Linguistics 18: Papers in Optimality Theory, GLSA, Amherst, MA, 249-384.

McCarthy, John J., Wendell Kimper \& Kevin Mullin (2012). Reduplication in Harmonic Serialism. Morphology 22:2, $173-232$.

Prince, Alan \& Paul Smolensky (1993/2004). Optimality Theory: Constraint Interaction in Generative Grammar. Blackwell Publishing, Malden, MA.

Riggle, Jason (2006). Infixing Reduplication in Pima and its Theoretical Consequences. Natural Language \& Linguistic Theory 24:3, 857-891.

Somerday, Megan (2015). (Some) Partial Reduplication is Full Reduplication. Bui, Thuy \& Deniz Özyıldız (eds.), NELS 45: Proceedings of the Forty-Fifth Annual Meeting of the North East Linguistic Society, GLSA, Amherst, MA, vol. 2, 79-92.

Steriade, Donca (1988). Reduplication and Syllable Transfer in Sanskrit and Elsewhere. Phonology Yearbook 5:1, 73155. 